\section{Sleep and Biological Rhythms}

Helga Peter

Marburg, Deutschland

\section{Definition}

Zeitschrift.

Siehe $\triangleright$ „Schlafmedizinische Fachgesellschaften, Fachzeitschriften und Publikationsforen“. 